\title{
Design, development and deployment of a hand/wrist exoskeleton for home-based rehabilitation after stroke - SCRIPT project
}

F. Amirabdollahian $\dagger, *$, S. Ates $\ddagger$, A. Basteris $\dagger$,

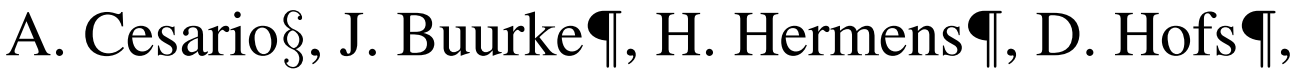
E. Johansson\|, G. Mountain ††, N. Nasr ††, S. Nijenhuis $\uparrow$, G. Prange $\llbracket, ~ N . ~ R a h m a n \dagger$, P. Sale $\S$, F. Schätzlein ††, B. van Schooten $\uparrow$ and A. Stienen $\ddagger$

\author{
$\dagger$ University of Hertfordshire, United Kingdom \\ $\ddagger$ University of Twente, the Netherlands \\ $\S$ San Raffaele Pisana, Italy \\ $\Phi$ Roessingh Research and Development, the Netherlands \\ \|User Interface Design, Germany \\ $\dagger \dagger$ University of Sheffield, Sheffield, UK
}

(Accepted July 29, 2014. First published online: September 23, 2014)

\section{SUMMARY}

Changes in world-wide population trends have provided new demands for new technologies in areas such as care and rehabilitation. Recent developments in the the field of robotics for neurorehabilitation have shown a range of evidence regarding usefulness of these technologies as a tool to augment traditional physiotherapy. Part of the appeal for these technologies is the possibility to place a rehabilitative tool in one's home, providing a chance for more frequent and accessible technologies for empowering individuals to be in charge of their therapy.

Objective: this manuscript introduces the Supervised Care and Rehabilitation Involving Personal Tele-robotics (SCRIPT) project. The main goal is to demonstrate design and development steps involved in a complex intervention, while examining feasibility of using an instrumented orthotic device for home-based rehabilitation after stroke.

Methods: the project uses a user-centred design methodology to develop a hand/wrist rehabilitation device for home-based therapy after stroke. The patient benefits from a dedicated user interface that allows them to receive feedback on exercise as well as communicating with the health-care professional. The health-care professional is able to use a dedicated interface to send/receive communications and remote-manage patient's exercise routine using provided performance benchmarks. Patients were involved in a feasibility study $(n=23)$ and were instructed to use the device and its interactive games for $180 \mathrm{~min}$ per week, around $30 \mathrm{~min}$ per day, for a period of 6 weeks, with a 2-months follow up. At the time of this study, only 12 of these patients have finished their 6 weeks trial plus 2 months follow up evaluation.

Results: with the "use feasibility" as objective, our results indicate 2 patients dropping out due to technical difficulty or lack of personal interests to continue. Our frequency of use results indicate that on average, patients used the SCRIPT1 device around 14 min of self-administered therapy a day. The group average for the system usability scale was around 69\% supporting system usability.

Conclusions: based on the preliminary results, it is evident that stroke patients were able to use the system in their homes. An average of 14 min a day engagement mediated via three interactive games is promising, given the chronic stage of stroke. During the 2nd year of the project, 6 additional games with more functional relevance in their interaction have been designed to allow for a more variant

* Corresponding author. E-mail: f.amirabdollahian2@herts.ac.uk 
context for interaction with the system, thus hoping to positively influence the exercise duration. The system usability was tested and provided supporting evidence for this parameter. Additional improvements to the system are planned based on formative feedback throughout the project and during the evaluations. These include a new orthosis that allows a more active control of the amount of assistance and resistance provided, thus aiming to provide a more challenging interaction.

KEYWORDS: Rehabilitation Technology; Home-based therapy systems; Stroke rehabilitation; Adaptive systems.

\section{Introduction}

Research into motor relearning and processes of cortical reorganisation after stroke have provided a neurophysiological basis for key aspects that stimulate restoration of arm function. ${ }^{1,2}$ These key aspects include high training intensity, active initiation and execution of movements, and application of functional exercises. With respect to the training intensity, repetition of movements has been shown to strengthen the representation of the trained movements in the brain. ${ }^{3}$ Training with a higher frequency or longer duration stimulates functional recovery of the arm. ${ }^{4-10}$ Concerning active initiation and execution of movements, brain studies have shown that cortical activity is larger during active execution of movements than during passive motion, predominantly in secondary motor area's and basal ganglia. ${ }^{11}$ Also, motor cortex excitability is higher after active movement training, accompanied by increased agonist activity and decreased antagonist activity, in contrast to passive movement training. ${ }^{12}$ Exercise therapy focusing on active initiation and execution of movements is associated with improved arm function. ${ }^{13-15}$ Regarding functional exercises, several studies have shown that functional training, focusing on activities of daily life that are relevant to the patient, results in a normalisation of brain activity ${ }^{1,7}$ that is related to improvements in motor control and functional abilities. ${ }^{16}$ Therefore, functional exercises are another important feature of exercise therapy to stimulate motor recovery after stroke. ${ }^{3}$

\subsection{Rehabilitation technology}

Technological innovations provided an opportunity to design interventions that take many key aspects for stimulation of motor relearning into account, of which rehabilitation robotics is a well-known example. With such a device, the required amount of support to the arm and hand movements can be provided, thereby allowing active practice of movements when this is not possible otherwise. This increases the potential to train intensively, with active contribution by the patient to functional exercises. The application of rehabilitation robotics has been shown to be effective. ${ }^{17-19}$ However, transfer of robotic training effects to activities in daily life is limited, as is observed for most interventions in stroke rehabilitation, including conventional therapy. ${ }^{4}$

Contemporary robot-aided therapy focuses mainly on the proximal arm, and often results in improvements in the proximal arm only, without generalisation to the wrist and hand. ${ }^{17}$ In order to maximise independent use of the upper extremity in daily life, it is important to include functional practice of the wrist and hand. Several technological interventions have been developed for distal arm training, such as the Bi-Manu-Track, which showed promising results while making intensive training of elbow and wrist possible. ${ }^{20}$ In addition, a distal trainer has been designed to complement the MIT-Manus robotic device for the proximal arm. ${ }^{21}$ The first preliminary results of the stand-alone use of this wrist module were optimistic. ${ }^{22}$ Moreover, initial research into alternating training of the proximal and distal arm suggests that early involvement of distal arm movements is favourable over proximal training alone in terms of transfer of treatment effects to the untrained arm segments. ${ }^{22}$ After stroke, especially wrist, hand and finger extension is problematic, and it is often the last symptom to show some improvement, if any, after recovery of leg function and proximal arm function. Therefore, it is expected that a training environment in which distal control for grasping and manipulation of objects is optimally supported has a large potential. Due to the inherent complexity of designing grasping tools, a smaller and more recent subset has targeted training of the hand and wrist (e.g. ${ }^{23-25}$ ).

\subsection{Training at home}

Besides this treatment benefit, one of the major advantages of technologically supported interventions is the potential to automate therapy, especially when a rehabilitation device incorporates a motivating training environment, for instance through interactive means, including (bio)feedback 
about the patient's performance. Regarding a virtual training environment, combining (bio)feedback with progressive exercises is essential to promote independent training with tele-supervision by professionals. Addition of augmented feedback to exercises can stimulate the learning process, by making patients more aware of their performance. ${ }^{26}$ Recent studies provide some insight into the optimal application of augmented feedback. A combination of augmented visual and sensory feedback is promising, ${ }^{27,28}$ as well as placing emphasis on movement errors to stimulate motor (re)learning. ${ }^{29,30}$

In combination with a motivating and progressive exercise environment (serious gaming), autonomy and continuity of treatment is enabled in a way that stimulates motor relearning, in the patient's home. Such application of rehabilitation devices allows a patient to train independently, in an intensive, active and functional way, in his/her own environment with continuous access to treatment facilities. This provides the patient with a sense of control and autonomy, which might also contribute to a better treatment outcome in itself. It also allows very efficient use of the treatment facilities, and enables practice in more efficient and effective ways. Distributed practice sessions and random variation within practice is more beneficial than blocked practice to improve arm functionality after stroke, due to a more active participation of the patient in the learning process. ${ }^{26}$ Moreover, spreading practice sessions across several days also results in enhancement of performance during the remaining practice sessions and on retention of the learned task compared with the same sessions practiced on one day. ${ }^{31}$ Thus ideally patients should be able to train variable exercises several (short) times a day, for most days of the week.

In addition, the use of such systems offers the possibility to quantify each patient's specific impairments and his/her progress during treatment, using sensitive and objective quantitative indicators of movement performance..$^{32}$ Furthermore, a computerised training environment including (bio)feedback enables remote monitoring of movements and progress (tele-monitoring) and remote supervision by the therapist (tele-supervision). This saves one-to-one treatment time and house visits, allows therapists to attend to multiple patients, increases productivity and alleviates the physical burden on the therapist, which in turn can relieve the pressure on today's healthcare system, where ageing of the population will result in fewer therapists and more patients.

This manuscript introduces the Supervised Care and Rehabilitation Involving Personal Telerobotics (SCRIPT) project. At the heart of the project is a system designed using a user-centred design methodology aimed at providing home-based rehabilitation for the wrist and fingers after stroke. The project focuses on robot-mediated intervention and benefits from specific interaction design primed to encourage longer interaction by using a therapeutic auto-tuning system and benefitting from interaction via games that provide a chance for fun and motivating interaction. Games are designed in a way to encourage specific gestures of the hand and wrist, while also focusing on more active participation of the patient in his/her own therapy. Two user interfaces, for the patient and for the healthcare professional in charge of the patient's therapy program, are developed to allow for remote management of the therapy as well as providing feedback to patients. Different aspects of design and development as well as feasibility evaluation results are detailed and discussed in the following sections.

\section{Design Methodology}

We employed an interdisciplinary research design to co-design and evaluate the SCRIPT system with people with stroke, carers and stroke professionals in three clinical sites in the United Kingdom (UK), the Netherlands (NL) and Italy (IT). The user-driven system development framework comprises of two main phases. In phase 1, we engaged with potential users of the SCRIPT system to create a clear picture of the target users and the context in which the system intends to operate. The aim of this phase was to provide the technology developers with a detailed knowledge of the diversity of users of the system. A mix of health and social sciences and design methods were used to collect data in this phase including focus groups, in-depth interviews and cultural probes. ${ }^{33}$

The findings of the focus groups, which showed participants' general attitude and feelings toward technology, were further explored and refined by conducting in-depth interviews and cultural probing during two successive home visits (Fig. 1). Cultural probe materials such as diaries and photography activities gave us an opportunity to observe participants' behaviours between two home visits whilst the outcome of the probing was used to prompt participants during the interviews. The findings of this phase were summed up to create a collection of persona-based scenarios ${ }^{34}$ as representatives of a range of users and their social and personal contexts, their experiences of stroke and technology use, 

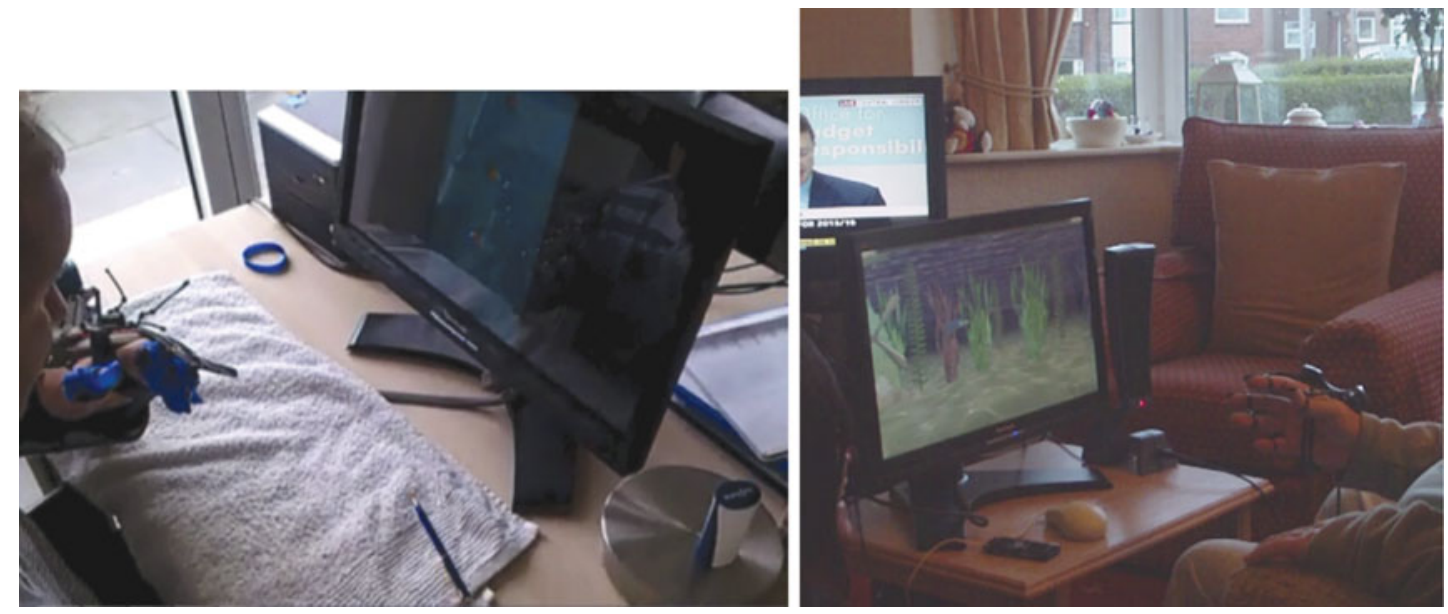

Fig. 1. (Colour online) Home visits with cultural probes and in-depth interviews.

their needs, activities and goals. These user models served to identify user requirements and informed design of the iterative prototyping.

In phase 2, the resulting prototypes were evaluated across three clinical sites using participatory formative evaluation methods such as cognitive walkthrough and cooperative evaluation. ${ }^{35}$ Different components of the SCRIPT system including the orthosis, user interface and games were tested out with the members of the steering group committees. In addition, the system was evaluated in participants' homes in which people with stroke and their carers were encouraged to think aloud while trying out the system in order to identify main usability problems. The identified problems were summarised and fed back into the process of design to improve the next iteration of the system. The resulting prototypes are tested, redesigned and developed in iterative cycles.

\section{Hardware, Software and System Architecture}

\subsection{Mechatronic device}

SCRIPT Prototype 1 (SP1) is a wrist, hand and finger orthosis that assists individuals after stroke that suffer from impairments caused by spasticity and abnormal synergies (Fig. 2). These impairments are characterised in the wrist and hand by excessive involuntary flexion torques that prevent the hand to be used for many to most activities in daily life. The SP1 can passively offset these undesired torques, but it cannot actively generate or control movements. The user needs to use voluntary muscle activation to perform movements and thus needs to have some residual muscle control to successfully use the device. A passive actuation mechanism is chosen so that devices are inherently safe and can be integrated into home-use easier.

The hardware components of the SP1 are:

- Physical interfaces to the user: forearm shell, hand plate, and digit caps with Velcro straps. These physical interfaces are the only components of the SP1 that come in contact with the user.

- Wrist-torque transfer mechanism: double parallelogram between forearm shell and hand plate that allows wrist flexion-extension but blocks all other wrist rotations.

- Torque-generation mechanisms: digit leaf springs and adjustable tension cords.

- Device controller: micro-controller board that reads the sensors and converts these readings to physiological relevant parameters and transfers them to the main unit. Arm support: commercially acquired arm support (Saebo MAS) that supports the arm against gravity to enable arm movements in individuals after stroke.

- State sensors: integrated measurements units (IMUs) with which the forearm posture can be estimated.

The SP1 physically interfaces with the forearm, hand and fingers of the users using respectively a forearm shell, a hand plate and individual digit caps. To guarantee safe and comfortable interaction, it 


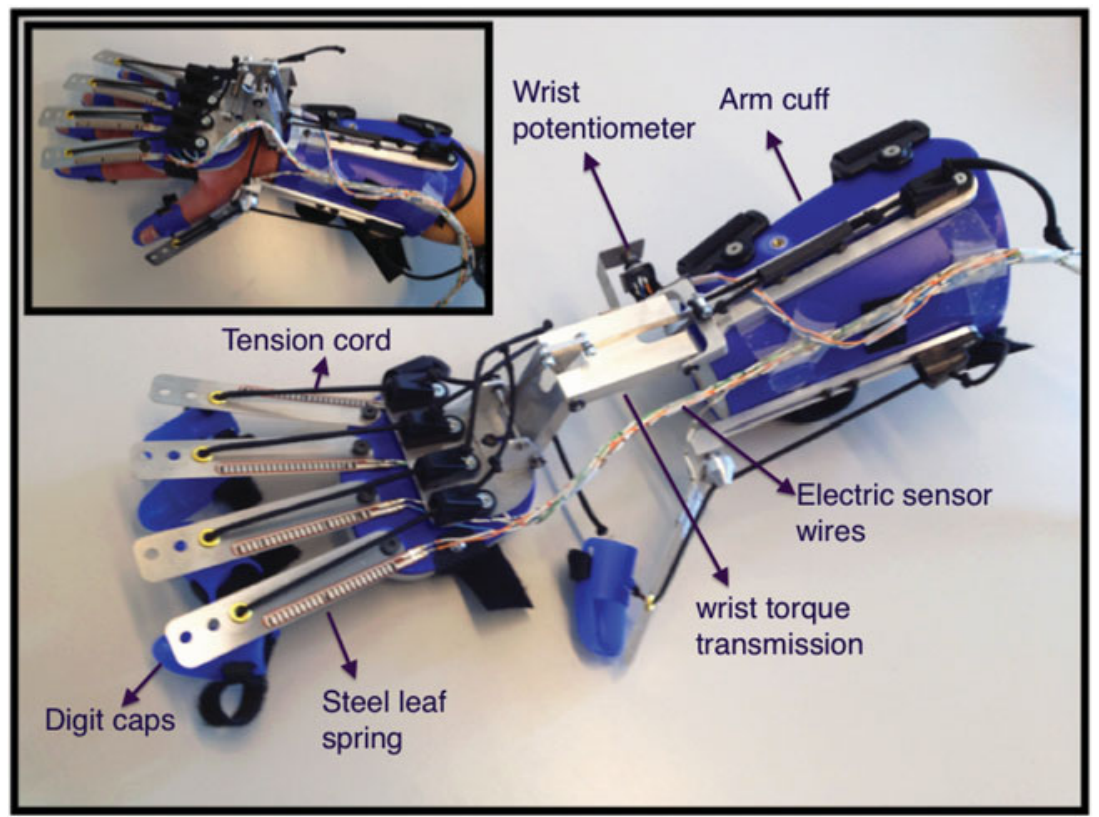

Fig. 2. (Colour online) SCRIPT Prototype 1. From left to right: blue digit caps, steel leaf springs, black finger tension cords, red/white/black bending sensor, blue hand plate, black tension-cord stops, metal wristtorque transmission, black wrist tension cords, blue forearm cuff. The thumb cap, leaf spring, tension cord and additional rotation joint are visible below the wrist joint. All the sensors are connected to the computer interface via electric sensor wires.

uses commercially available physical interfaces with a proven track record from Saebo Inc. (Charlotte, NC, USA). The Saebo forearm shells, hand plates and caps are available in multiple sizes and for both left and right hands, that are needed to custom fit the SP1 to a wide range of body dimensions.

The SP1 applies the external extension torques on the fingers via passive leaf springs and elastic tension cords. The leaf springs allow the extension force to be applied perpendicular to the fingertip for most of the range of motion, but cannot be directly attached to the finger due to the misaligned digit axes of the finger and the device. The leaf spring has cord guides through which the tension cord is routed and is covered in a shrink-wrap to protect the patients against its sharp edges. The tension cord is used to give the finger freedom of movement relative to the leaf springs. The cord is also used to adjust the amount of support by tensioning it more or less using the tension-cord stops on the top of the hand plate.

The force generation and application mechanisms for the thumb are identical to the ones for the fingers. To allow additional freedom of movement in the thumb needed for thumb opposition, the thumb mechanisms has an additional rotational degree of freedom that coincides with the wrist axis, with any misalignment being allowed for by the flexibility of the tension cords.

The wrist mechanism uses a double parallelogram between forearm shell and hand plate that allows wrist flexion-extension but blocks all other wrist rotations. The double parallelogram is needed to prevent misalignment between human and device axes and makes the device comfortable to use. Through the parallelogram, the rotation of the hand around the wrist flexion-extension axis of the wrist is transferred to the parallelogram clamp at the forearm. There this rotation is actuated using an elastic tension cord. The tension in the cord can be adjusted using the cord stops at the elbow end of the forearm shell.

The external extension force in the digits and the wrist can be adjusted by tensioning the elastic cords. The digits and the wrist have a single tension cord each, and each cord has multiple knots on it. These knots can be clamped in the cord stops on the device and tensioning the cord will produce more external extension force. The user is instructed to change the tension based on his impairment and his therapy progress via the physical therapist and the graphical user interface.

The SP1 closely follows the contours of the human body. Most components stay within a volume less than $30 \mathrm{~mm}$ away from the body. The wrist parallelogram, needed to allow wrist flexion/extension, 
requires $75 \mathrm{~mm}$ from the body at maximum wrist extension. The device is not overly disruptive when used as a therapy tool, but it is too bulky to be a permanent aid for daily use.

To improve patient comfort, all sharp edges of the devices are either sanded down or covered using protective material such as plastic shrink wrappers. All electrical components (sensors and wirings) are covered using cable sleeves.

The SP1 is equipped with sensors to measure the joint rotations and the applied external extension torques. On the digits this is realised by measuring the deflection of the leaf spring using flexible bending sensors. This deflection can be converted to both an angular deflection of the digit and the applied extension force using the known stiffness properties of the leaf spring. The integrated bending sensors in the leaf springs at the fingers are good enough to differentiate between different grasps ${ }^{36}$ and can detect small changes of motion, but are only able to resolve between large absolute angle deviations of roughly 20 degrees. ${ }^{37}$ These limitation are caused not by the mechanism itself, but by the type of bending sensor used: the flexible resistance-based sensors suffer from natural decay in step response that makes it hard to get accurate absolute values from the sensors. In the wrist, the flexion-extension axis rotation is measured using a potentiometer at the forearm link of the parallelogram.

Finally, the hand position in global space is approximated using the UM6 ultra-miniature orientation sensor (Integrated Measurement Unit) from CH Robotics placed on the forearm that uses rate gyros, accelerometers, magnetic sensors, and an onboard 32-bit ARM Cortex processor to compute sensor orientation. The orientation and acceleration of the forearm are used for the estimation of slow and fast movements and movement directions.

In order to extract MCP, PIP, and DIP rotations from a single measurement with the help of one bending sensor, a simple angle estimation algorithm is derived. Each flex sensor provides one bending angle reading to each individual finger. In order to extract each digits angular position (MCP, PIP, DIP rotations) from one sensor reading, some assumptions have to be made. It is assumed that there is no dead zone with the flex sensors and rotation of each phalanx is linear and covers the full range of motion except the distal phalanx. Finger caps overlap distal phalanx and middle phalanx at the same time and limit the range of motion of distal phalanx drastically. Abduction and adduction movements are neglected, as well. In addition, a simple initialisation/calibration procedure is required to measure the maximum and minimum sensor values. A similar approach can be easily applied to thumb and wrist.

The bending sensors at the leaf springs and the potentiometer at the wrist mechanisms are sampled using the analog-digital converters in the Arduino Nano microprocessor board. The Arduino passes on the sampled values to the main PC using a Universal Serial Bus (USB) connection. The IMU is connected to a serial-to-usb converter and again connected to the main PC using a USB connection. For both, custom Windows 7 drivers were written to make the signals available to the connected software.

\subsection{User interfaces}

The interface of the SCRIPT system consists of two separate parts: a user interface (UI) for the patient (Patient UI) and a UI for the health care professionals (HCP UI). Two separate concepts were developed since the parts are targeted for different user groups, performing different tasks. Also the choice of the platform required a different design, as the Patient UI is based on a touch screen and the HCP UI is controlled with keyboard and mouse using a conventional display.

\subsection{User interface for the health care professional}

An important screen for the HCP UI is the patient overview (Fig. 3), which is presented directly after login. The table lists all patients, and presents an overview of the current state of the patients' training:

- Patient's name, age and week of therapy

- The development of the game scores, using simple colour coding (red, yellow, green)

- Patient's own rating of current condition, using a simple colour coding and smileys

- The latest training session

- The total length of this week's training sessions

- New messages received from the patient 


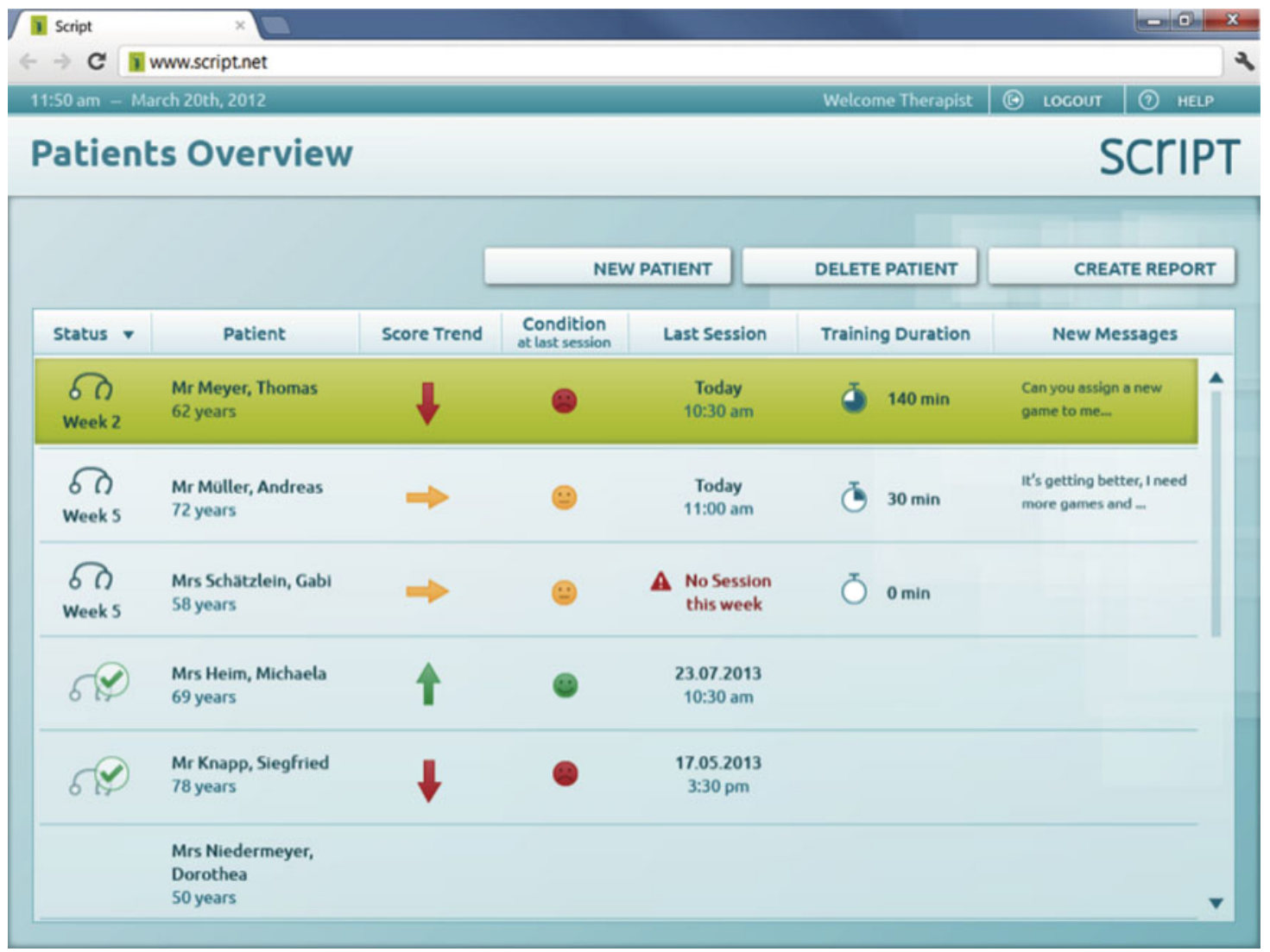

Fig. 3. (Colour online) Health care professionals' user interface - patient overview screen.

In this overview the therapist can immediately recognise patients that need further care, e.g. due to a patent's negative condition rating. A double-click on a cell will lead the HCP directly to the corresponding page, where he has access to detailed information and functionalities. To work with a patient, the HCP UI consists of four main areas:

1. Messages: The message area allows the therapist to exchange text or audio messages with the patients.

2. Progress: The progress area consists of several pages with different charts, e.g. active range of motion (aROM) in-Game (Fig. 4), aROM during Calibration, Move Count, Training Duration, Training Session and Game Score. This area provides the therapist with a detailed feedback on the progress of the patient, using visualisations.

3. Games: The games area contains the basic functionality for the therapy with SCRIPT. In this area the therapist assigns games to the patients and adapts the difficulty level of the game. The difficulty set here relates to selection of game levels requiring more difficult to achieve gestures, for example game difficulty increases when lateral movement of the arm are added to the flexion and extension of the hand. This follows a system similar to Gentile's taxonomy of motor skills where Stationary actions are classified as simplest skills while In Motion functions are thought to require more complex skills. ${ }^{38}$

4. Condition: The content and design of the "condition" area is currently under progress, In this area the therapist will receive feedback on the status of the patient (presented on the left hand side of Fig. 4) in terms of affected side and diagnosis as well as other performance metrics allowing them to set the therapy plan with these considerations.

\subsection{User interface for the patient}

To ensure consistency between the Patient and HCP UI, the same design concept was applied to both interfaces. Also, the areas in the patient UI are mainly the same as in the HCP UI, but with 


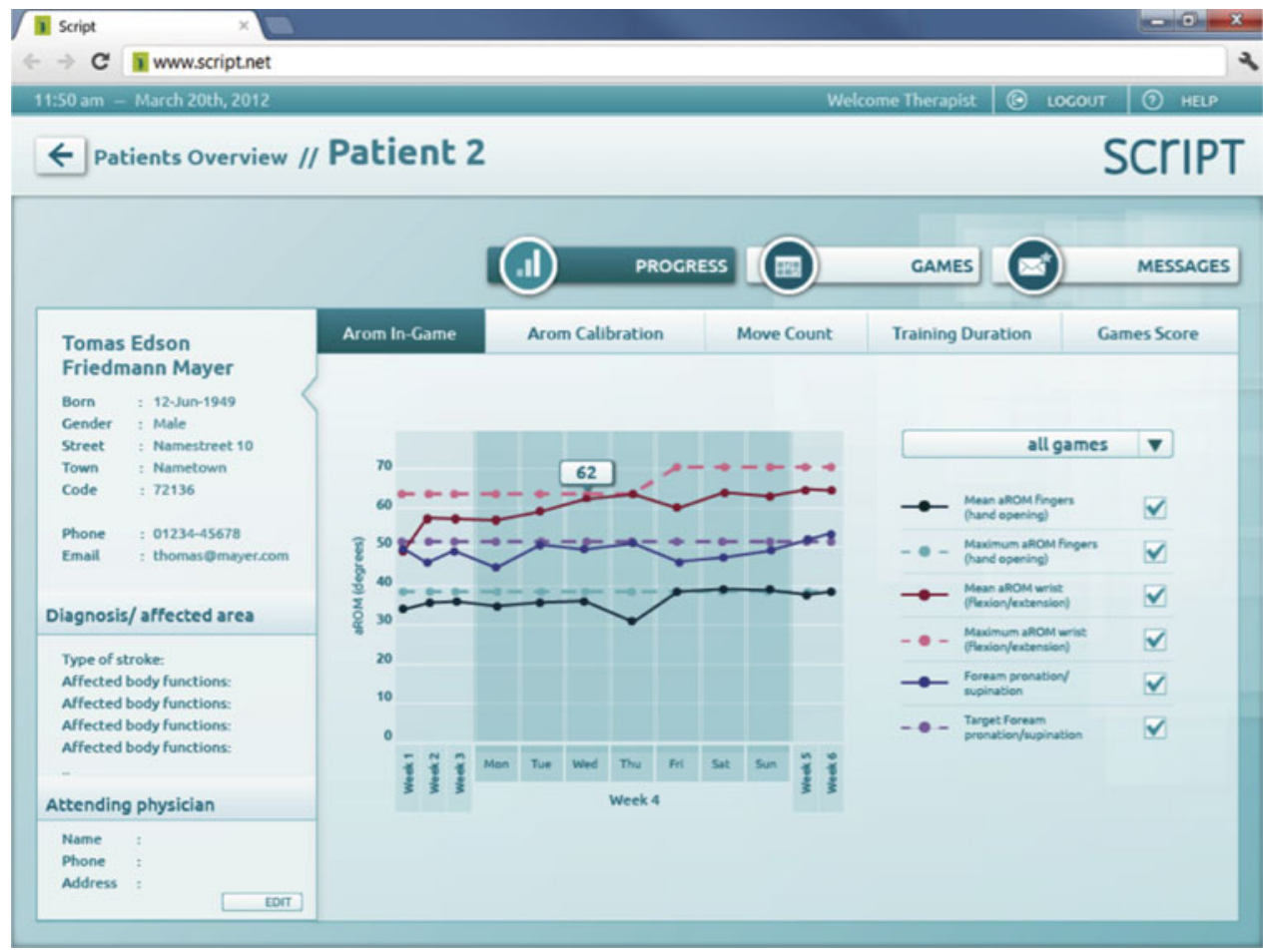

Fig. 4. (Colour online) Patient progress shown in aROM tab highlighting changes in active range of motion during game play.

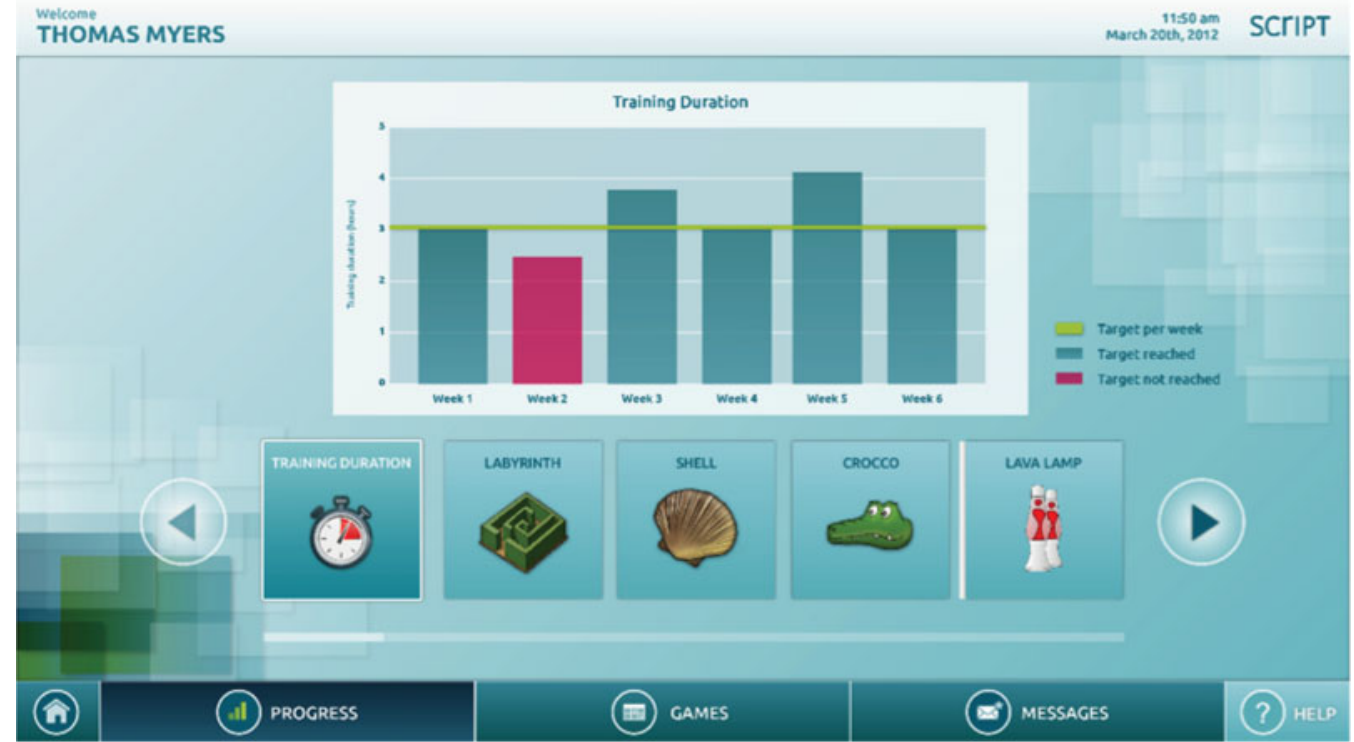

Fig. 5. (Colour online) Progress screen highlighting progress across the 6 weeks of study.

relevant content important to patients. A requirement for the Patient UI was to offer quick access to the recommended games and to be experienced as "easy to use" by the patients. Due to these requirements, the UI provides direct access to the games on the home screen after login. Patient UI consists of three main areas:

1. Progress: In this section (Fig. 5) the patient can check the training progress, e.g. game scores and training duration. Scientific statistics like the aROM are not needed for the patient. Design of the charts is adapted to the target users and target system, by reducing the amount of information 


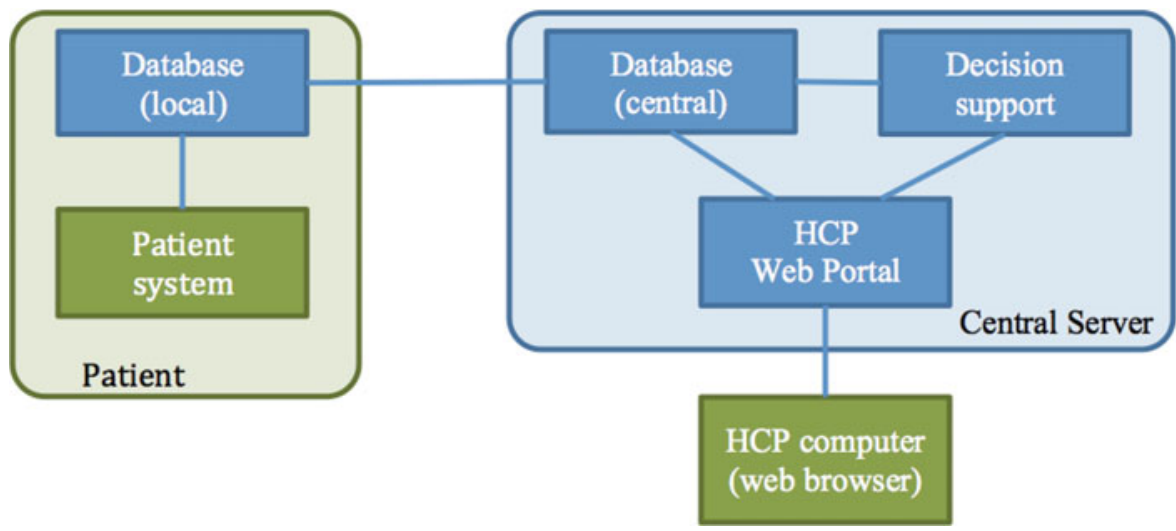

Fig. 6. (Colour online) Tele-robotic support platform and its components. The platform comprises of the blue components.

and increasing the size of the interaction elements. Motivational messages are displayed within and after the games, to motivate the patients to continue with the training. Example messages are "Maybe you can come back for more practice soon?", "Target time has been accomplished", "You have just finished training for $\mathrm{N}$ minutes", "N minutes more to reach your target this week".

2. The patient can choose between different games, assigned by the therapist. For each game, detailed information on how to play the game is available to the patient.

3. Messages: As for the HCP UI, the patient can exchange textual or audio messages with the therapist. The patient enters the message using a touch screen keyboard.

\subsection{Tele-robotic support platform}

The tele-robotic support platform concerns the implementation of remote access to the patient's system. This enables HCPs to create and change exercise plans during the therapy, to check on the patient data and progress, and communicate with the patient. The platform consists of three components: a replicated database, a HCP webportal, and a decision support system. See Fig. 6.

The patient's computer communicates with a central server through a replicated database. On the central server there is a webportal and a decision support system. HCPs can access the data through the webportal. Authentication for both patients and HCPs is performed through a regular username-password combination. All network communication is secured through SSL.

3.5.1. Database (remote management interface). Communication between the patient computer and the central system proceeds entirely through a replicated database, implemented in MySQL. The database is accessed locally through an XML-RPC API. When a new patient system is deployed, as 9 prototype systems are re-used, the replication process wipes any old data and loads essential data like the patient's information and the initial exercise plans from the central server. From then on, the patient system can function autonomously, but will sync data when an Internet connection is available. It replicates only the data belonging to that patient, so that privacy of other patients is ensured. The local database also has a development mode, which allows functioning without a central server altogether.

\subsection{Adaptive and therapeutic human-robot interaction}

An important goal of the project is its focus on adaptive and therapeutic human robot interaction (THRI). The objective here is to cater for inter-individual differences sensed in interaction. To make this possible, a dedicated component is placed in the software architecture (see Fig. 8), which constitutes a middleware between the orthosis and the games. Indeed, the THRI processes the signal generated from the device and delivers to the games information about movement's performance of the subject. Such information can be of analog type, as angular or positional coordinate of a joint, or discrete, as the message that a specific pattern of movement (gesture) has just been performed. Gestures currently available relate to hand state (open, close or in a grasping position), or the wrist (extended, flexed, prone or supine). Also, we included the recognition of gross arm movements 

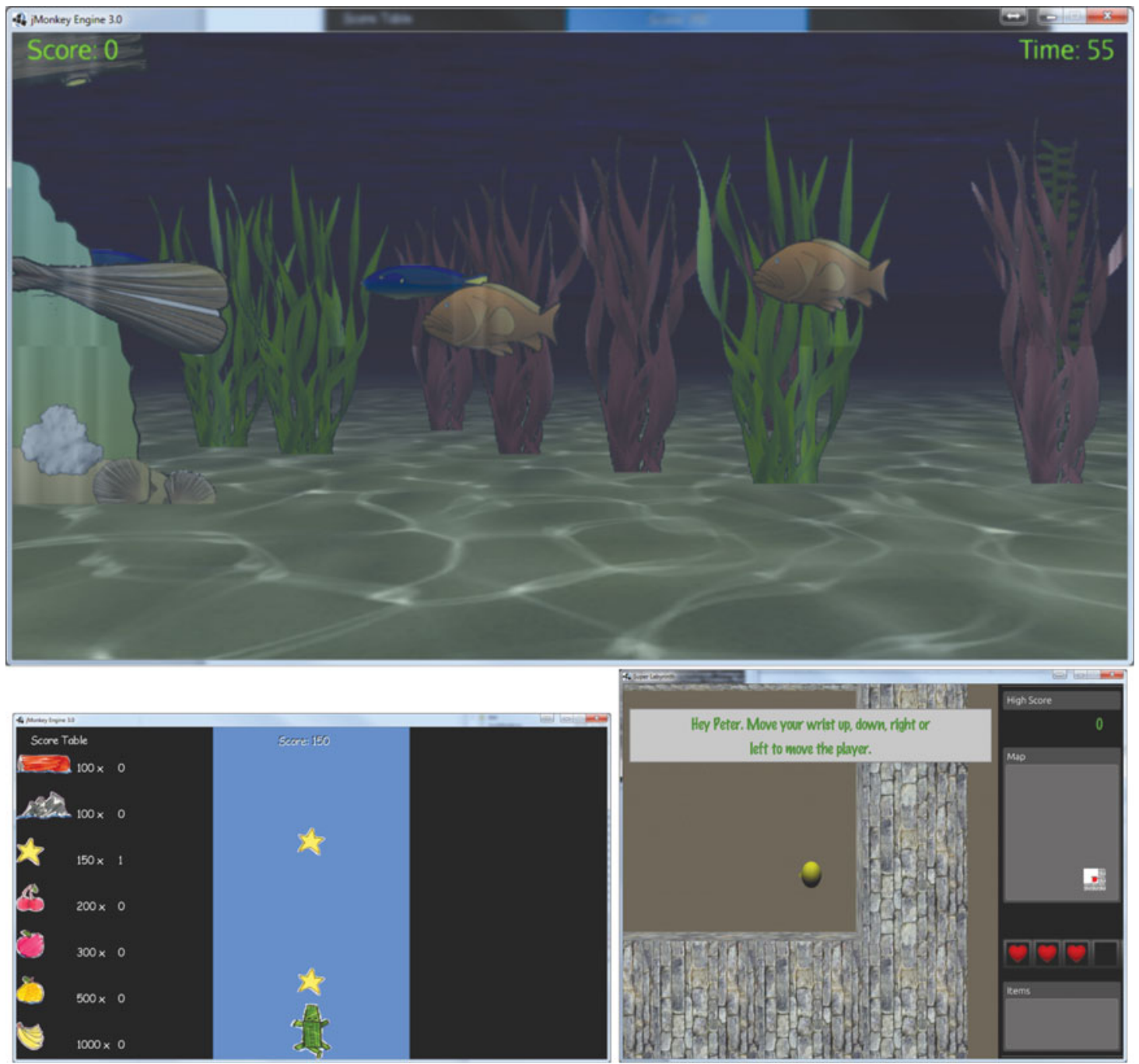

Fig. 7. (Colour online) Three prototype exercise games, top: Sea Shell; bottom left: Super Crocco; bottom right: Labyrinth.

(antero-posterior and lateral displacement of the hand) as those normally performed during activities of daily living by using accelerometers on the forearm part of the arm-cuff.

These movements are matched with actions within the games that are intended for providing therapeutic exercise. Three games were designed and tested within years 1 and 2 of the project: "Sea Shell", "Super Crocco" and "Labyrinth" as shown in Fig. 7.

In the Sea Shell game, the patient operates a shell by his/her hand in order to catch fishes. In the Super Crocco game, in addition to grasping, wrist flexion and extension are performed to avoid obstacles, and lateral movements of the hand to move the character on the screen. The Labyrinth game offers, in addition to this, training of forearm prone/supination and antero-posterior movements of the hand.

One of the major challenges in performing gesture recognition with stroke survivors is the interindividual variability in motor symptoms. For instance, while normative data about range of motion (ROM) for the upper limb joints is available for healthy subjects, ${ }^{39,40}$ each patient constitutes a per se case. Considering this, we developed a calibration procedure which measures range of motion and duration of movements. ${ }^{41}$ In a preliminary analysis of the data from seven subjects, we showed that such a procedure was effective in $85 \%$ of the attempts (1044 out of 1229 tests) with durations differing across movements of up to $30 \mathrm{~s}$ for hand opening. ${ }^{42}$ This procedure, potentially exploitable for other various movement signals, is run ahead of any practice sessions for the movements which will be required within the game. In this way, the gesture recognition is tailored on the individual patient 
capabilities. For instance, wrist extension is marked by reaching the $90 \%$ of the ROM measured during the calibration. This approach has been effective for all the 23 patients included. In total, the calibration procedure has been successful in $88 \%$ of the attempts (3791 out of 4298). Considering as an instance wrist flexion/extension, median values ranged across subjects from 0.4 to approximately 135 degrees (97\% of the device ROM). Overall, considering all subjects involved in the study, the gesture recognition has identified 558449 movements.

Another requirement for the therapeutic human robot interaction is to be adaptive. In the domain of rehabilitation robotics, a fundamental principle is that the robot should "assist-as-needed". ${ }^{43}$ However, the passive device does not allow any automatic regulation of the physical support provided, which is set by the therapist. Hence, the THRI further modulates the task difficulty using the speed of the game to prolong interaction time.

At the beginning, movements are required at a speed matching the time measured during the calibration. At the end of each movement, patient's performance is assessed by considering whether he/she is lagging or leading with respect to a model-generated movement profile ${ }^{44}$ which matches their own movements in terms of amplitude and duration. The lag/lead score is the fraction of trajectory in which the subject was anticipating the reference trajectory. The speed of the game is then rescaled based on the last $10 \mathrm{lag} / \mathrm{lead}$ scores, in order to make the exercise nor too challenging nor too easy, according to the challenge point framework. ${ }^{45}$ Future development will include the support for the active-actuated device to allow for changing assistance/resistance, recognition of functional type of gestures and an alternative method to estimate the subject's contribution to movement, based on the energy flow between the patient and the device.

\subsection{Architecture}

The system has a wide range of software which needs to meet very different requirements, such as distributed fault-tolerant communication, real-time control and monitoring of the devices and an intuitive, responsive, and graphically rich therapeutic user-interface. The system adopts a modular component-based architecture, as shown in Fig. 8, which supports software that is scalable, reusable, lightweight, dynamically reconfigurable and portable between platforms. The architecture allows flexibility in terms of choice of operating system, programming languages, tools and libraries. The architecture is open and flexible, but at the same time clearly defines the interfaces, which facilitate distributed system development with periodic integration cycles.

The patient's system runs on Microsoft Windows 7 Professional, as this meets the needs of being responsive to user actions, while at the same time providing a rich graphical interface and simplifying the design and development of software. Windows 7 also provides a rich set of communications and data storage mechanisms. It does not offer deterministic timings, but experimental results have shown that, coupled with a multi-core PC and sufficient memory, it is adequate for the performance requirements of SCRIPT. The therapist's system can run on Windows and Linux systems.

Software components are implemented as independent co-operating processes, using a variety of programming languages and tools, such as MySQL, Apache, C++, Python, PHP, Java, Javascript and JMonkey. The SCRIPT project uses Google Protocol Buffers to define and manipulate message data sent using stream and datagram sockets between software components on the patient PC. Protocol Buffers provide a flexible, efficient and automated mechanism for serialising structured data in C++, Java and Python. Using protocol buffer is lighter weight than manipulating XML data structures and allows changes to message data structures without breaking existing code. Communication between the patient PC and the remote server is via XML-RPC.

\section{Experimental Validaiton}

Although it is assumed that patients will practice independently when provided with the opportunity, this is a highly relevant question when deploying a technology-supported system for training of the arm and hand in the home setting. Therefore, this is one of the main issues addressed during evaluation and impact assessment of the SCRIPT system.

\subsection{Feasibility evaluation}

Besides Formative Evaluation (FE) focussing on user-engagement in design and system development to enhance the acceptability and usability of the system, the clinical study design applied in this 


\section{SCRIPT SYSTEM ARCHITECTURE (OVERVIEW, SCHEMATIC)}

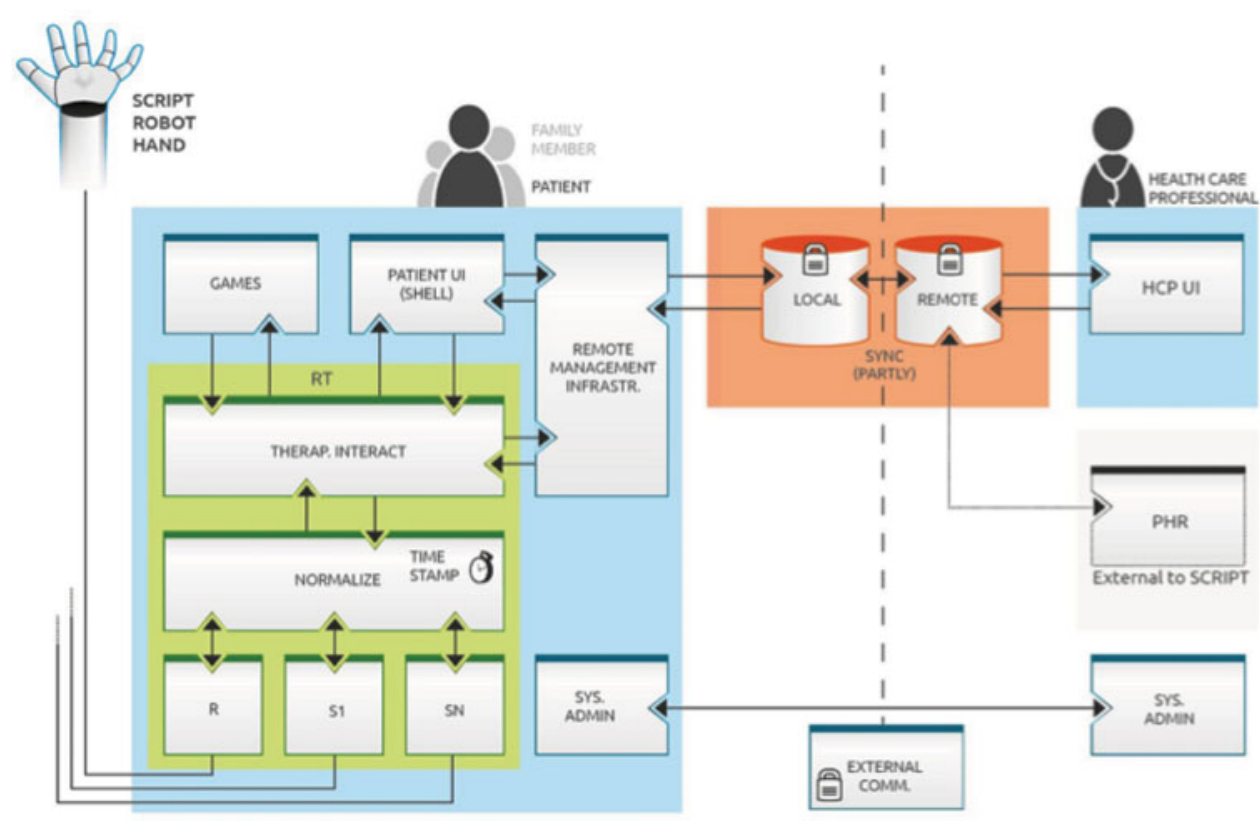

Fig. 8. (Colour online) SCRIPT System Architecture, low-level system components include actuator (R), sensors on the orthosis (S1) and external sensors $(\mathrm{SN})$.

project (Summative Evaluation (SE)) involves evaluation of the SCRIPT system in a situation where treatment protocols haven't been fixed or personally supervised. On the contrary, except for the recommendation to practice 30 min per day for 6 days a week, participating chronic stroke patients are left entirely free in their choice on when and for how long they want to practice with the SCRIPT system during the 6 weeks deployment at their home. During these 6 weeks, patients are remotely supervised on their progress by a healthcare professional (HCP) through the HCP portal. The progress in terms of game scores serves as the basis for the HCP to select appropriate exercises targeting e.g., wrist flexion/extension movements combined with grasping/hand opening gestures. This decision is refined, if needed, during short weekly home visits by the HCP to check on the patient.

The summative evaluation assesses feasibility of the self-administered telerobotic devices on several levels across three European-wide user-evaluation centres (University of Sheffield in the United Kingdom, San Raffaele S.p.A. in Italy, Roessingh Research and Development in the Netherlands), which allows validation of effectiveness of the SCRIPT system. In terms of user acceptance, actual amount of use, usability and patient's motivation are examined during and after the use period.

\subsection{Results}

To date, 23 of the intended 30 chronic stroke patients have been included (see Table 1), who had to be between 6 and 60 months post-stroke and display at least $15^{\circ}$ of active elbow flexion and a quarter range of active finger flexion. Of these, data for 12 patients is available from baseline and post-training evaluations, conducted 1 week before and then after the 6-week training period. Seven male and five female chronic stroke patients were included, with mean age of 54 years ( \pm 12 years) and mean time post stroke of 11 months ( \pm 6 months). The group involved 25\% mildly, 25\% moderately and $50 \%$ severely affected stroke patients, based on a categorisation of initial Fugl-Meyer (FM) score. ${ }^{46}$

Among the first 12 patients were two dropouts, because of their inability to work with the SCRIPT system due to technical problems, or no desire to work with the SCRIPT system anymore. The preliminary findings of the ongoing study suggest that deployment of self-administered training is feasible, as reflected in actual use of the system by the majority of the patients, as monitored in terms of training duration. The actual amount of training, measured using system logs created during game play, was on average $94.4( \pm 43.6)$ minutes/week across the first 10 patients (ranging from 34 to 
Table I. Subject characteristics and outcomes before and after training.

\begin{tabular}{lcc}
\hline Characteristics and outcomes & Mean $( \pm \mathrm{SD})$ & Range \\
\hline Gender (male/female)* & $7 / 5$ & \\
Stroke severity (mild/moderate/severe)* & $3 / 3 / 6$ & \\
Age (years) & $54( \pm 12)$ & $32-69$ \\
Time post stroke (months) & $11( \pm 6)$ & $6-26$ \\
Training duration (minutes per week) & $94.4( \pm 43.6)$ & $34-166$ \\
System Usability Scale (0-100\%) & $69.3( \pm 20.0)$ & $38-100$ \\
\hline
\end{tabular}

* absolute numbers

166 min per week between patients), which comes down to an average of 14 min of self-administered training each day for 6 weeks. The group average of the System Usability Scale was $69.3( \pm 20.0)$, indicating that usability of the SCRIPT system is promising, with a good chance of acceptance in the field ${ }^{47}$ This further supports the feasibility of the SCRIPT system to be used independently for training at home so far.

\section{Discussion}

The present findings suggest that independent home-based training with the SCRIPT system is feasible. Patients are choosing to exercise by themselves for about 15 min a day for 6 weeks on average. It has to be noted that this is without external motivation such as fixed appointments with healthcare professionals for online remote supervision during exercise, which distinguishes the SCRIPT application from recent telerehabilitation approaches in stroke. ${ }^{48}$ Moreover, this type of training so far shows potential to enhance sensorimotor function of the affected arm. Due to large variations observed across patients, specific attention needs to be paid to relations between outcome measures and patient characteristics on an individual basis (as illustrated in Figure) in ongoing work. However, the present research is the first exploration of independent arm/hand training at home using a physical hand/wrist orthosis combined with remote monitoring (telerehabilitation). Ongoing analysis of the clinical outcomes, as well as the amount of use, intensity of training, usability and user acceptance of the complete sample allows for a comprehensive analysis of relations between these characteristics. Early results from these evaluations are presented in a subsequent paper showing larger variations amongst patients concerning clinical gains when using the SCRIPT system. ${ }^{49}$ With such information, the group of patients that might benefit most from this type of intervention may be identified. One particular question of interest concerns the relation between the amount of use and clinical changes, to explore whether patients will actually use a telerehabilitation system provided to them and whether more use will actually result in larger clinical gains. This will inform our future developments.

\section{Conclusions and Future Work}

The design, development and deployment of a home-based rehabilitation device for mediating repetitive exercise via a passive-actuated hand/wrist exoskeleton device was presented here. Aspects highlighted included the importance of user-centred design and inclusion of potential users as codevelopers and co-designers. This was made possible by employing multiple phases of interaction during the design cycles. A critical step here was to start with understanding the potential users and their diversity using in-depth interviews and cultural probes. These culminated in system requirements that were used for developing the prototype system. The prototype was then assessed against those user requirements using formative evaluation cycles often consisting of focus groups and steering group meetings.

This manuscript highlights different aspects of development, ranging from the hardware including the mechatronic device, to software components such as patient and health care professional user interfaces.The objective of this evaluation was to assess the feasibility of a home-based rehabilitation device. Although early, preliminary results obtained from 12 patients that participated in this evaluation phase indicate supportive evidence for feasibility of such devices for home use. 
Future work focuses on active-tuning of the amount of assistance provided to the hand and wrist, while providing an additional six exercise games with a more versatile set of gestures incorporated within the new games. This is to enhance the functional and goal-oriented nature of the exercises. Also, the decision support system under development will provide a chance to assist in monitoring patients' remotely using performance benchmarks and by detecting sudden and slow changes in progress. A second summative evaluation phase is planned to test the resulting system.

\section{Acknowledgements}

This work has been partially funded under Grant FP7-ICT-288698(SCRIPT) of the European Community Seventh Framework Programme. We are grateful to SCRIPT consortium for their ongoing commitment and dedication to the project and to a large number of stroke patients, their families and healthcare professionals that have provided us with formative and summative feedback during the development of this project.

\section{References}

1. J. D. Schaechter, "Motor rehabilitation and brain plasticity after hemiparetic stroke," Prog. Neurobiology 73(1), 61-72 (2004).

2. J. W. Krakauer, “Arm function after stroke: From physiology to recovery," Semin. Neurology 25(4), 384-395 (2005).

3. B. E. Fisher and K. J. Sullivan, "Activity-dependent factors affecting poststroke functional outcomes," Top. Stroke Rehabil. 8(3), 31-44 (2001).

4. R. C. Wagenaar and O. G. Meijer, "Effects of stroke rehabilitation: A critical review of the literature," Rehabil. Sci. 4(3), 61-73 (1991).

5. G. Kwakkel, R. C. Wagenaar, T. W. Koelman, G. J. Lankhorst and J. C. Koetsier, "Effects of intensity of rehabilitation after stroke a research synthesis," Stroke 28(8), 1550-1556 (1997).

6. G. Kwakkel, R. C. Wagenaar, J. W. R. Twisk, G. J. Lankhorst and J. C. Koetsier, "Intensity of leg and arm training after primary middle-cerebral-artery stroke: a randomised trial," Lancet 354(9174), 191-196 (1999).

7. G. Nelles, W. Jentzen, M. Jueptner, S. Müller and H. C. Diener, "Arm training induced brain plasticity in stroke studied with serial positron emission tomography," Neuroimage 13(6), 1146-1154 (2001).

8. T. Platz, "Evidence-based arm rehabilitation-a systematic review of the literature," Der Nervenarzt 74(10), 841 (2003).

9. G. Kwakkel, R. van Peppen, R. C. Wagenaar, S. W. Dauphinee, C. Richards, A. Ashburn, K. Miller, N. Lincoln, C. Partridge, I. Wellwood, et al. "Effects of augmented exercise therapy time after stroke a meta-analysis," Stroke 35(11), 2529-2539 (2004).

10. A. C. Lo, P. D. Guarino, L. G. Richards, J. K. Haselkorn, G. F. Wittenberg, D. G. Federman, R. J. Ringer, T. H. Wagner, H. I. Krebs, B. T. Volpe, et al. "Robot-assisted therapy for long-term upper-limb impairment after stroke," New England J. Med. 362(19), 1772-1783 (2010).

11. C. Weiller, M. Jüptner, S. Fellows, M. Rijntjes, G. Leonhardt, S. Kiebel, S. Müller, H. C. Diener and A. F. Thilmann, "Brain representation of active and passive movements," Neuroimage 4(2), 105-110 (1996).

12. A. Kaelin-Lang, L. Sawaki and L. G. Cohen, "Role of voluntary drive in encoding an elementary motor memory," J. Neurophysiol. 93(2), 1099-1103 (2005).

13. H. M. Feys, W. J. De Weerdt, B. E. Selz, G. A. C. Steck, R. Spichiger, L. E. Vereeck, K. D. Putman and G. A. Van Hoydonck, "Effect of a therapeutic intervention for the hemiplegic upper limb in the acute phase after stroke a single-blind, randomized, controlled multicenter trial," Stroke 29(4), 785-792 (1998).

14. S. Barreca, S. L. Wolf, S. Fasoli and R. Bohannon, "Treatment interventions for the paretic upper limb of stroke survivors: A critical review," Neurorehabilitation Neural Repair 17(4), 220-226 (2003).

15. L. E. Kahn, P. S. Lum, W. Z. Rymer and D. J. Reinkensmeyer, "Robot-assisted movement training for the stroke-impaired arm: Does it matter what the robot does?," J. Rehabil. Res. Dev. 43(5), 619 (2006).

16. A. Feydy, R. Carlier, A. Roby-Brami, B. Bussel, F. Cazalis, L. Pierot, Y. Burnod and M. A. Maier, "Longitudinal study of motor recovery after stroke recruitment and focusing of brain activation," Stroke 33(6), 1610-1617 (2002).

17. G. B. Prange, M. J. A. Jannink, C. G. M. Groothuis-Oudshoorn, H. J. Hermens and M. J. IJzerman, "Systematic review of the effect of robot-aided therapy on recovery of the hemiparetic arm after stroke," $J$. Rehabil. Res. Dev. 43(2), 171 (2006).

18. G. Kwakkel, B. J. Kollen and H. I. Krebs, "Effects of robot-assisted therapy on upper limb recovery after stroke: A systematic review," Neurorehabilitation Neural Repair 22(2), 111-121 (2008).

19. J. Mehrholz, T. Platz, J. Kugler and M. Pohl, "Electromechanical and robot-assisted arm training for improving arm function and activities of daily living after stroke," Stroke 40(5), e392-e393 (2009). 
20. S. Hesse, G. Schulte-Tigges, M. Konrad, A. Bardeleben and C. Werner, "Robot-assisted arm trainer for the passive and active practice of bilateral forearm and wrist movements in hemiparetic subjects," Arch. Phys. Med. Rehabil. 84(6), 915-920 (2003).

21. H. I. Krebs, J. Celestino, D. Williams, M. Ferraro, B. Volpe and N. Hogan, "A Wrist Extension for MitManus," In: Advances in Rehabilitation Robotics (Springer Berlin Heidelberg, Germany, 2004) pp. 377-390.

22. H. I. Krebs, B. T. Volpe, D. Williams, J. Celestino, S. K. Charles, D. Lynch and N. Hogan, "Robot-aided neurorehabilitation: A robot for wrist rehabilitation," IEEE Trans. Neural Syst. Rehabil. Eng. 15(3), 327-335 (2007).

23. L. Masia, H. I. Krebs, P. Cappa and N. Hogan, "Design and characterization of hand module for whole-arm rehabilitation following stroke," IEEE/ASME Trans. Mechatronics 12(4), 399-407 (2007).

24. R. C. V. Loureiro, B. Lamperd, C. Collin and W. S. Harwin, "Reach \& Grasp Therapy: Effects of the gentle/g System Assessing Sub-Acute Stroke Whole-Arm Rehabilitation," IEEE International Conference on Rehabilitation Robotics, 2009. ICORR 2009, IEEE (2009) pp. 755-760.

25. E. Carmeli, S. Peleg, G. Bartur, E. Elbo and J.-J. Vatine, "Handtutortm enhanced hand rehabilitation after stroke?a pilot study," Physiotherapy Res. Int. 16(4), 191-200 (2011).

26. C. J. Winstein and J. C. Stewart, "Conditions of task practice for individuals with neurologic impairments," Textbook Neural Repair Rehabil. 2, 89-102 (2006).

27. B. I. Molier, E. H. F. Van Asseldonk, H. J. Hermens and M. J. A. Jannink, "Nature, timing, frequency and type of augmented feedback; does it influence motor relearning of the hemiparetic arm after stroke? a systematic review," Disability \& Rehabil. 32(22), 1799-1809 (2010).

28. B. I. Molier, Influence of Augmented Feedback on Learning Upper Extremity Tasks After Stroke (University of Twente, the Netherlands, 2012).

29. J. L. Patton and F. A. Mussa-Ivaldi, "Robot-assisted adaptive training: Custom force fields for teaching movement patterns," IEEE Trans. Biomed. Eng. 51(4), 636-646 (2004).

30. E. H. F. van Asseldonk, M. Wessels, A. H. A. Stienen, F. C. T. van der Helm and H. van der Kooij, "Influence of haptic guidance in learning a novel visuomotor task," J. Physiol.-Paris 103(3), 276-285 (2009).

31. C. H. Shea, Q. Lai, C. Black and J.-H. Park, "Spacing practice sessions across days benefits the learning of motor skills," Hum. Mov. Sci. 19(5), 737-760 (2000).

32. H. I. Krebs, B. T. Volpe, M. Ferraro, S. Fasoli, J. Palazzolo, B. Rohrer, L. Edelstein, N. Hogan, et al. "Robotaided neurorehabilitation: From evidence-based to science-based rehabilitation," Top. Stroke Rehabil. 8(4), 54-70 (2002).

33. B. Gaver, T. Dunne and E. Pacenti, "Design: cultural probes," Interactions 6(1), 21-29 (1999).

34. A. Cooper, R. Reimann and D. Cronin, About face 3: The Essentials of Interaction Design (John Wiley \& Sons, Wiley Publishing Inc, Indianapolis, USA, 2012).

35. A. Monk, L. Davenport, J. Haber and P. Wright, Improving your Human-Computer Interface: A Practical Technique (Prentice Hall London, 1993).

36. B. Leon, A. Basteris and F. Amirabdollahian, "Comparing Recognition Methods to Identify Different Types of Grasps for Hand Rehabilitation," 7th International Conference on Advances in Computer-Human Interactions. (ACHI2014) (2014) pp. 109-114.

37. S. Ates, J. Lobo-Prat, P. Lammertse, H. van der Kooij and A. H. Stienen "Script Passive Orthosis: Design and Technical Evaluation of the Wrist and Hand Orthosis for Rehabilitation Training at Home. IEEE... International Conference on Rehabilitation Robotics:[proceedings] (2013) pp. 1-6.

38. R. A. Magill and D. I. Anderson, Motor Learning and Control: Concepts and Applications, Vol. 11 (McGraw-Hill, New York, 2007).

39. D. J. Magermans, E. K. J. Chadwick, H. E. J. Veeger and F. C. T. Van Der Helm, "Requirements for upper extremity motions during activities of daily living," Clinical Biomechanics 20(6), 591-599 (2005).

40. C. J van Andel, N. Wolterbeek, C. A. M. Doorenbosch, DirkJan H. E. J. Veeger and J. Harlaar, "Complete 3d kinematics of upper extremity functional tasks," Gait Posture 27(1), 120-127 (2008).

41. J. L. Emken, J. E. Bobrow and D. J. Reinkensmeyer, "Adaptive human-robot interaction based on lag-lead mod- elling for home-based stroke rehabilitation," IEEE International Conference on Systems, Man and Cybernetics (SMC2013) (IEEE, 2013).

42. A. Basteris and F. Amirabdollahian, "Rapid Assessment of Range of Motion and Movement Duration During Human-Robot Interaction," World Congress for NeuroRehabilitation (WCNR) 2014, Istanbul, Turkey (Apr. 8-12, 2014).

43. J. L. Emken, J. E. Bobrow and D. J. Reinkensmeyer, "Robotic Movement Training as an Optimization Problem: Designing a Controller that Assists Only as Needed," Proceedings of the 9th International Conference on Rehabilitation Robotics, 2005. ICORR 2005, (IEEE, 2005) pp. 307-312.

44. R. Chemuturi, F. Amirabdollahian and K. Dautenhahn, "Adaptive training algorithm for robot-assisted upper-arm rehabilitation, applicable to individualised and therapeutic human-robot interaction," $J$. Neuroengineering Rehabil. 10(1), 102 (2013).

45. M. A. Guadagnoli and T. D. Lee, "Challenge point: A framework for conceptualizing the effects of various practice conditions in motor learning," J. Motor Behav. 36(2), 212-224 (2004).

46. A. R. Luft, S. McCombe-Waller, J. Whitall, L. W. Forrester, R. Macko, J. D. Sorkin, J. B. Schulz, A. P. Goldberg and D. F. Hanley, "Repetitive bilateral arm training and motor cortex activation in chronic stroke: a randomized controlled trial," Jama 292(15), 1853-1861 (2004). 
47. A. Bangor, P. Kortum and J. Miller, "Determining what individual sus scores mean: Adding an adjective rating scale," J. Usability Stud. 4(3), 114-123 (2009).

48. F. Coupar, A. Pollock, L. A. Legg, C. Sackley and P. van Vliet, "Home-based therapy programmes for upper limb functional recovery after stroke," Cochrane Database Syst Rev. (2012). doi: 10.1002/14651858.CD006755.pub2.

49. G. B. Prange, S. M. Nijenhuis, P. Sale, A. Cesario, N. Nasr, G. Mountain, F. Amirabdollahian and J. H. Buurke, "Preliminary Findings of Feasibility and Compliance of Technology-Supported Distal Arm Training at Home after Stroke," In: Replace, Repair, Restore, Relieve - Bridging Clinical and Engineering Solutions in Neurorehabilitation (W. Jensen, O. K. Andersen and M. Akay, eds.) (Springer International Publishing, Berlin, 2014) pp. 665-673. 